\title{
A Three-Dimensional Model of the Human Atria With Heterogeneous Wall Thickness and Fibre Transmurallity - A Realistic Platform for the Study of Atrial Fibrillation
}

\author{
Sara Rocher ${ }^{1}$, Laura Martínez ${ }^{1}$, Alejandro Lopez ${ }^{1}$, Ana Ferrer ${ }^{1}$, Damián Sánchez-Quintana ${ }^{2}$, Javier \\ Saiz ${ }^{1}$ \\ ${ }^{1}$ Centro de Investigación e Innovación en Bioingeniería, Univeristat Politècnica de \\ València,Valencia, Spain \\ ${ }^{2}$ Departamento de Anatomía, Universidad de Extremadura, Badajoz, Spain
}

\begin{abstract}
Multiscale cardiac modelling has been increasingly used since it provides a promising framework to improve our understanding of atrial fibrillation physiopathology.

In this study, we present a highly detailed threedimensional model of the human atria including heterogenous wall thickness and fibre transmurality, suggested as relevant factor in arrhythmogenic dynamics.

We hypothesized that the structural definition of computer models influences the electrical propagation and to test it we compared the fibrillatory activity of the new model with two models less detailed anatomically but with the same electrical and tissular properties. The three studied models generated different arrhythmic patterns, with the appearance of re-entrant drivers at different atrial regions.

We conclude that it is very important to use complete realistic atrial models, especially for atrial fibrillation studies, to obtain reliable results. Additionally, the developed model allows to consider new variables of study involving atrial wall thickness transmurality.
\end{abstract}

\section{Introduction}

Atrial Fibrillation (AF) is the most common supraventricular arrythmia, with a growing prevalence with aging population. It is considered a major cause of morbidity and mortality and is associated with increased risk of stroke, impaired quality of life and high healthcare related expenses [1].

Despite the progress in medical technology, the available treatments are still suboptimal and have modest efficacy, in part due to the incomplete understanding of the physiopathology of the disease. AF burden has powered the development of computational modelling in cardiac electrophysiology and simulations have been increasingly used for the study of atrial activity under variable conditions.

The rapid increase in the amount of experimental data and computational resources have allowed great advances in cardiac modelling. Several generic atrial models have already been published incorporating important features, such as morphological, histological and functional information [2][3][4]. However, to the best of our knowledge, the atrial wall thickness and fibre transmurality, suggested as important features in reentrant dynamics [5][6], have not been taken into account.

The objective of this work is to develop a highly detailed three-dimensional (3D) model of the human atria and to study if the structural definition of the models affects propagation during AF.

\section{Methods}

\subsection{Geometrical Model}

We developed a new atrial model by improving our previous 3D model of the human atria [4]. The mesh was divided into 21 regions and subdivided into 56 subregions for a detailed anatomical and fiber orientation description.

In the last years, the atrial structural details have been analysed as it can help to improve the safety and the success rate of ablation treatments. We reviewed measurements of atrial wall thickness (AWT) heterogeneity from different techniques: post-mortem studies [7], Magnetic Resonance Imaging (MRI) [8] and Computed Tomography [9]. The AWT of the right atrium (RA) is very heterogenous because of the presence of the Crista Terminalis (CT) and the pectinate muscles (PM). The $\mathrm{CT}$ is the thickest region with a thickness of 5-8 mm while the thinnest part is the vestibule of the tricuspid valve (TV) with a mean thickness of $2 \mathrm{~mm}$. The AWT of the left atrium (LA) is much more uniform and measures on average $3 \mathrm{~mm}$. The thickest region is the anterior wall 
(LAW) with 4-5 $\mathrm{mm}$, while the thinnest region is the vestibule of the mitral valve (MV), with a mean thickness of $2 \mathrm{~mm}$. We defined the AWT in the new model by moving the epicardium and the endocardium surface nodes with a semi-automatic custom-made MATLAB routine. A manual smoothing was needed to remove abrupt changes between adjacent regions.

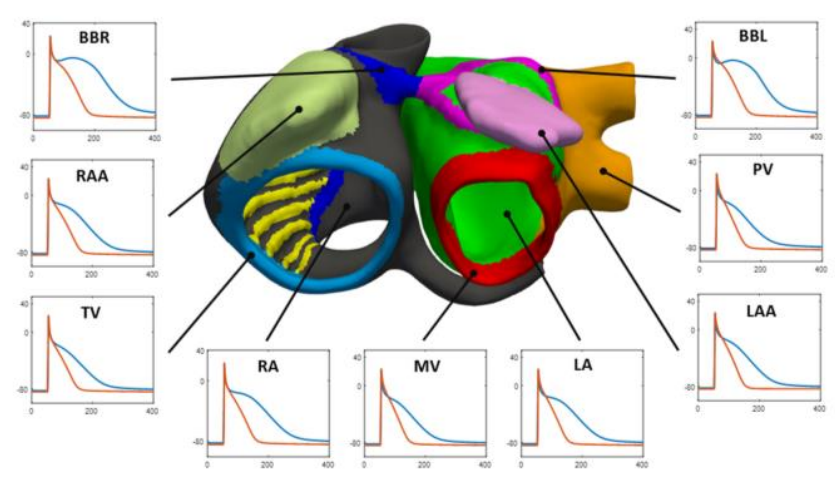

Figure 1. Action potentials produced by each of the nine variations of the $\mathrm{CRN}$ model at their corresponding atrial regions in control (blue) and PeAF (red). BBR: Right Buchman Bundle, LAA: Left Atrial Appendage, PV: Pulmonary Vein, BBL: Left Buchman Bundle.

The architecture of myocyte fibers has a major impact on the electrical propagation of the model. In human models, fiber orientation is usually analyzed from ex-vivo histological information [5] but recently diffusion tensor MRI images have also been proposed [10]. The RA has a complex fibrous structure, characterized by the abundant PMs, the prominent CT and a large appendage (RAA). In contrast, the LA has a smooth uniform appearance but presents marked transmural variations. The anterior and posterior (LPW) walls have a bi-layer structure with fibers from epicardium to endocardium running nearly perpendicular. We assigned the same fiber orientation as in our previous model [4] except for the elements belonging to the LAW and LPW, where from the midwall to the inner surface we rotated the epicardial fiber vector $45^{\circ}$ in the y-axis.

To obtain the volumetric model we meshed the atrial surface with hexahedral elements with spatial resolution of $300 \mu \mathrm{m}$ using the MeshGems-Hexa software.

\subsection{Electrical Model}

Electrophysiological and tissue properties are determinant for obtaining realistic simulations. In this study, the cellular electrical activity is modelled by the Courtemanche-Ramírez-Nattel (CRN) ionic model [11]. To reproduce the experimental heterogeneity in action potential (AP) morphology and duration of the different atrial regions we defined nine cellular models by adjusting the maximum conductance of the ionic channels $\mathrm{I}_{\mathrm{to}}, \mathrm{I}_{\mathrm{CaL}}, \mathrm{I}_{\mathrm{Kr}}, \mathrm{I}_{\mathrm{Ks}}$ and $\mathrm{I}_{\mathrm{K} 1}$ (Figure 1), similarly to other computational studies [4] [12]. Additionally, tissue heterogeneity was modelled by tuning in each region the longitudinal conductivities and the anisotropy ratios, to match the activation sequence of experimental data [13].

In permanent $\mathrm{AF}$ (PeAF) the recurrent fibrillation episodes force the cardiovascular system to develop mechanisms of adaptation. The electrical remodelling was introduced through the variation of the maximum conductance of $\mathrm{I}_{\mathrm{to}}, \mathrm{I}_{\mathrm{CaL}}, \mathrm{I}_{\mathrm{K} 1}, \mathrm{I}_{\mathrm{Kur}}, \mathrm{I}_{\mathrm{Ks}}, \mathrm{I}_{\mathrm{NaCa}}$ and $\mathrm{I}_{\mathrm{KACh}}$, similarly to [12]. Action potential duration (APD) shortening was within the range of experimental observations with a reduction of about 50\%. Gap junctional remodelling was also added with $15 \%$ reduced intracellular conductivity.

We stabilized the electrical models to smooth differences between neighbouring regions by applying to the sinoatrial node (SAN) 20 continuous beats with a basic cycle length (BCL) of $1000 \mathrm{~ms}$ in control simulations and $500 \mathrm{~ms}$ in PeAF simulations, $2 \mathrm{~ms}$ of duration and $28 \mathrm{pA} / \mathrm{pF}$ of amplitude. To validate the model, we applied a single stimulus to the SAN after the stabilization. To generate re-entrant activity, after the 20 pulses, we paced the coronary sinus (CS) using a continuous high frequency train $(\mathrm{BCL}=100 \mathrm{~ms}$ ) for 6 seconds.

The electrical propagation was solved by the monodomain formalism using the operator splitting numerical scheme with ELVIRA solver [14] with a constant time step of $0.01 \mathrm{~ms}$. The simulations were run on a computing node with sixty-four 16-core Intel $\circledast$ Xeon® Gold 6130 clocked at $2.10 \mathrm{GHz}$.

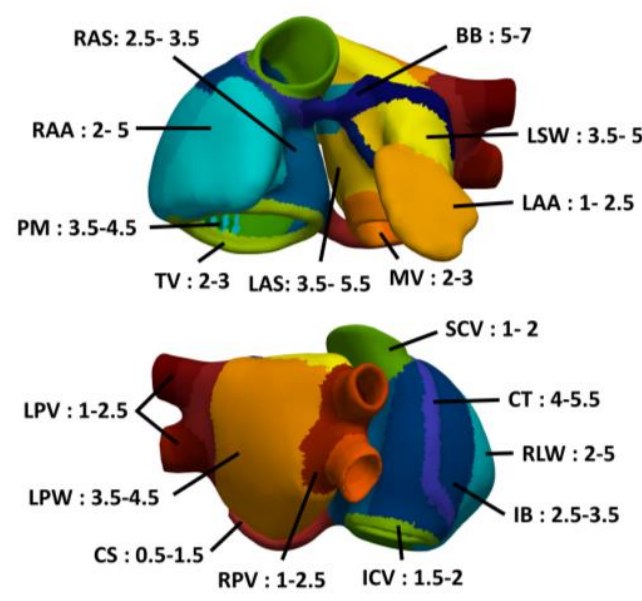

Figure 2. Atrial Wall Thickness ranges in $\mathrm{mm}$ at the different atrial regions. RAS: Right Atrial Septum, LAS: Left Atrial Septum, LSW: Left Superior Wall, BB: Bachman Bundle, LPV: Left Pulmonary Vein, RPV: Right Pulmonary Vein, RLW: Right Lateral Wall, IB: Intercaval Bundle, SCV: Superior Cava Vein, ICV: Inferior Cava Vein. 


\subsection{Phase Maps analysis}

In order to prove if the structural definition of the model affects propagation during $\mathrm{AF}$ simulations we compared the new 3D atrial model with two models less detailed: a model with heterogenous AWT but without transmurality in fibre orientation, our previous model with homogenous AWT [4].

Phase analysis is considered a useful descriptor to track the re-entrant dynamics. Through color-codes maps, it is possible to analyse temporal and spatial phase evolution. First we bandpass filtered $(8-12 \mathrm{~Hz})$ the atrial voltage membrane $(\mathrm{Vm})$ in order to allow rotor trip tracking, as in [12]. Then, we applied the Hilbert transform to the filtered $\mathrm{Vm}$ and phase maps were calculated. Finally, the phase singularity points (PS) were computed to track the rotor's trajectory on the atrial surface. For each element in the mesh and for all simulations time steps we computed the spatial variation of phase among its four nodes. The elements were designated as PS when the summed phase variation along the close path of the four nodes composing the element was $\pm 2 \pi$.

\section{Results and Discussion}

\subsection{D Atrial Model}

The new 3D model has 1.945.101 hexahedral elements and 2.174.034 nodes. The RA presents a mean thickness of $3 \mathrm{~mm}$ and the LA is slightly thicker with $3,4 \mathrm{~mm}$. (Figure 2). The ranges of resultant thickness values are consistent with the reviewed experimental data
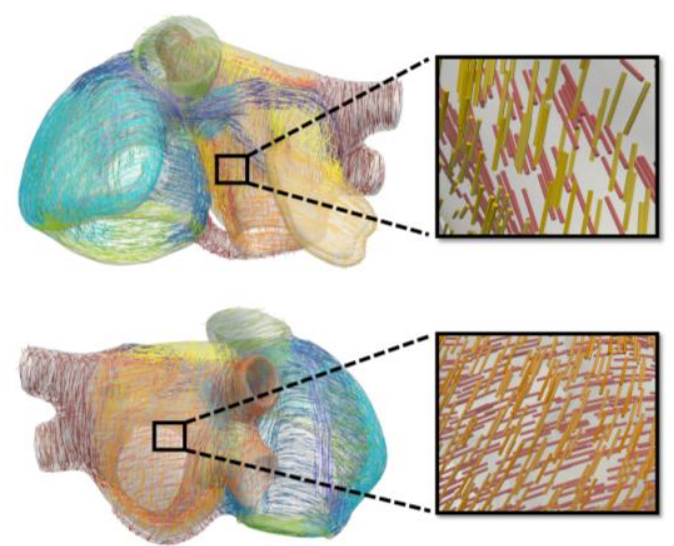

Figure 3. Fibre orientation of the different atrial regions with zoomed bilayer structure at LAW and LPW.

Atrial fibre orientations are shown in Figure 3. LAW epicardial fibres are aligned parallel to the RAS while in the endocardium they run from the BB to the MV. LPW epicardial fibres descend perpendicularly from the LSW to the MV while in the endocardium they are oriented mainly circumferentially from the RPV to the LPV.

We validated the model by comparing the activation sequence from the control simulation in sinus rhythm with experimental activation times measured by Lemery et al. [13]. Simulation starts with the SAN activation at $\mathrm{t}=0$ and continues with the depolarization of the RA. The $\mathrm{TV}$ is the last RA region activated at $\mathrm{t}=90 \mathrm{~ms}$ (experimental: $87 \pm 25 \mathrm{~ms}$ ). The propagation moves from the RA to the LA through the fossa ovalis and the BB, with the first activation at $\mathrm{t}=44 \mathrm{~ms}$ (experimental: $52 \pm 15$ $\mathrm{ms})$. The last activation takes place at the left inferior pulmonary vein at $\mathrm{t}=134 \mathrm{~ms}$ (experimental: $116 \pm 18 \mathrm{~ms}$ ).
(1)

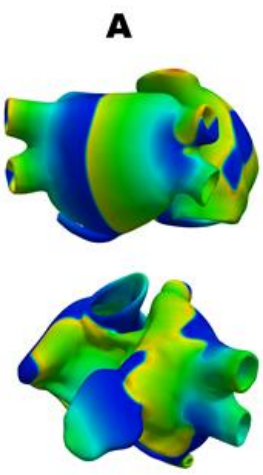

(3)

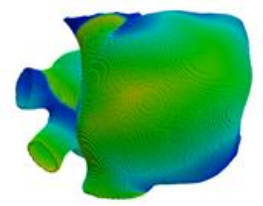

B

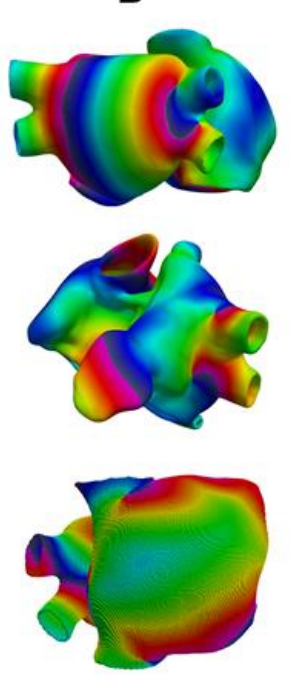

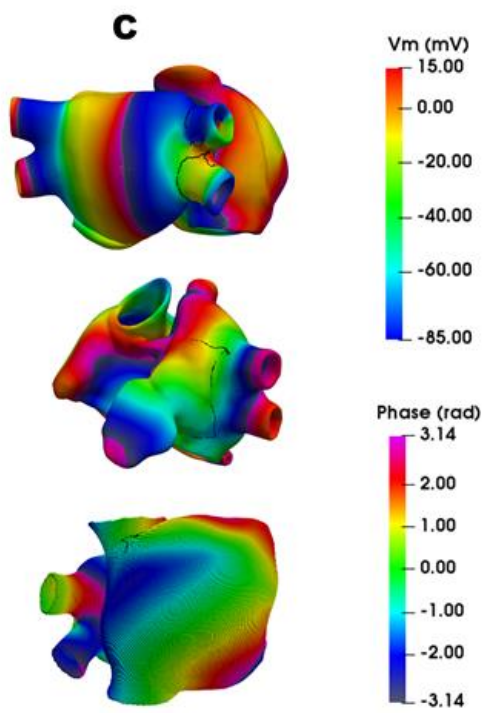

Figure 4. Fibrillatory Patterns. A) Simulated Vm in the new 3D model at $t=5155 \mathrm{~ms}$ (1), in the model with AWT but without fibre transmurality at $\mathrm{t}=2995 \mathrm{~ms}(2)$ and in our previous model at $\mathrm{t}=3160 \mathrm{~ms}$ (3). B) Computed phase maps at the same instants. C) In black, PSs tracking during the whole simulation over the phase maps. 


\subsection{Simulated fibrillation patterns}

The three studied models are able to reproduce fibrillatory activity with the presence of re-entrant drivers. However, in each model the arrhythmic propagation results in very different AF patterns. In the new model, we observe two rotors colliding on the LPW and moving circumferentially around the RPVs. In the model with anatomical AWT but without fibre transmurality, we also observe two rotors but on the anterior part of the LPVs and moving with a very unstable trajectory. In our previous model, it appears a stable single rotor on the SCV.

Only by adding heterogeneous AWT to the model the rotor appearance moved from the RA to the LA, demonstrating that AWT influences the initiation and perpetuation of arrythmias, as previously suggested in [6]. As expected, the addition of the bilayer structure in the LAW and the LPW also affects the AF pattern, since fibre orientation has a major impact in electrical propagation, especially in abnormal rhythms.

\section{Conclusions}

The presented highly detailed 3D multi-scale model of the human atria has proved to be a powerful platform for analysing both physiological and pathological conditions.

We demonstrate that the structural properties defined in the model affects atrial propagation, highlighting the need of using complete realistic computer models to obtain reliable results. The developed model is a promising tool to progress in the evaluation and treatment of arrythmias and allows to consider new variables of study involving atrial wall thickness transmurality, such as fibrosis or ablation lesions.

\section{Acknowledgments}

This work was partially supported by funding sources from Generalitat Valenciana ACIF/2018/174 and Programa Prometeu de la Conselleria d'Educació, Formació I Ocupació de la Generalitat Valenciana, award number PROMETEU/2016/088.

\section{References}

[1] J. Andrade, P. Khairy, D. Dobrev, and S. Nattel, "The clinical profile and pathophysiology of atrial fibrillation: Relationships among clinical features, epidemiology, and mechanisms," Circ. Res., vol. 114, no. 9 , pp. $1453-1468,2014$.

[2] G. Seemann, C. Höper, F. B. Sachse, O. Dössel, A. V. Holden, and H. Zhang, "Heterogeneous threedimensional anatomical and electrophysiological model of human atria," Philos. Trans. R. Soc. A Math. Phys.
Eng. Sci., vol. 364, no. 1843, pp. 1465-1481, 2006.

[3] O. V. Aslanidi et al., "3D virtual human atria: A computational platform for studying clinical atrial fibrillation," Prog. Biophys. Mol. Biol., vol. 107, no. 1, pp. 156-168, 2011.

[4] A. Ferrer et al., "Detailed anatomical and electrophysiological models of human atria and torso for the simulation of atrial activation," PLoS One, vol. 10, no. 11, pp. 1-29, 2015.

[5] S. Y. Ho and D. Sánchez-Quintana, "The importance of atrial structure and fibers," Clin. Anat., vol. 22, no. 1, pp. 52-63, 2009.

[6] A. Roy, M. Varela, and O. Aslanidi, "Image-based computational evaluation of the competing effect of atrial wall thickness and fibrosis on re-entrant drivers for atrial arrhythmias," Comput. Cardiol. (2010)., vol. 44, no. October, pp. 1-4, 2017.

[7] D. Sánchez-Quintana, G. Pizarro, J. R. López-Mínguez, S. Y. Ho, and J. A. Cabrera, "Standardized review of atrial anatomy for cardiac electrophysiologists," $J$. Cardiovasc. Transl. Res., vol. 6, no. 2, pp. 124-144, 2013.

[8] M. Varela et al., "Novel MRI Technique Enables NonInvasive Measurement of Atrial Wall Thickness," IEEE Trans. Med. Imaging, vol. 36, no. 8, pp. 1607-1614, 2017.

[9] R. Karim et al., "Algorithms for left atrial wall segmentation and thickness - Evaluation on an opensource CT and MRI image database," Med. Image Anal., vol. 50, pp. 36-53, 2018.

[10] F. Pashakhanloo et al., "Myofiber architecture of the human atria as revealed by submillimeter diffusion tensor imaging," Circ. Arrhythmia Electrophysiol., vol. 9 , no. 4, 2016

[11] M. Courtemanche, R. J. Ramirez, and S. Nattel, "Ionic mechanisms underlying human atrial action potential properties: Insights from a mathematical model," Am. J. Physiol. - Hear. Circ. Physiol., vol. 275, no. 1 44-1, 1998.

[12] L. Martinez-Mateu et al., "Factors affecting basket catheter detection of real and phantom rotors in the atria: A computational study," PLoS Comput. Biol. vol. 14, no. 3, pp. 1-26, 2018.

[13] R. Lemery et al., "Normal atrial activation and voltage during sinus rhythm in the human heart: An endocardial and epicardial mapping study in patients with a history of atrial fibrillation," $J$. Cardiovasc. Electrophysiol., vol. 18, no. 4, pp. 402-408, 2007.

[14] E. A. Heidenreich, J. M. Ferrero, M. Doblaré, and J. F. Rodríguez, "Adaptive macro finite elements for the numerical solution of monodomain equations in cardiac electrophysiology," Ann. Biomed. Eng., vol. 38, no. 7, pp. 2331-2345, 2010.

Address for correspondence:

Francisco Javier Saiz Rodríguez

Universitat Politècnica de València

Centro de Investigación e Innovación en Bioingeniería

Camino de Vera s/n, Edificio 8B

jsaiz@ci2b.upv.es 